Relations industrielles

Industrial Relations

\title{
Le licenciement en droit polonais
}

\section{Marek Pliszkiewicz}

Volume 36, numéro 1, 1981

URI : https://id.erudit.org/iderudit/029136ar

DOI : https://doi.org/10.7202/029136ar

Aller au sommaire du numéro

Éditeur(s)

Département des relations industrielles de l'Université Laval

ISSN

0034-379X (imprimé)

1703-8138 (numérique)

Découvrir la revue

Citer cet article

Pliszkiewicz, M. (1981). Le licenciement en droit polonais. Relations

industrielles / Industrial Relations, 36(1), 251-259.

https://doi.org/10.7202/029136ar

Tous droits réservés @ C Département des relations industrielles de l'Université Laval, 1981
Ce document est protégé par la loi sur le droit d'auteur. L'utilisation des services d'Érudit (y compris la reproduction) est assujettie à sa politique d'utilisation que vous pouvez consulter en ligne.

https://apropos.erudit.org/fr/usagers/politique-dutilisation/ 


\section{DROIT DU TRAVAIL}

\section{Le licenciement en droit polonais}

\section{Marek Pliszkiewicz}

\section{REMARQUES PRÉLIMINAIRES}

Il faut d'abord préciser la place du licenciement parmi tous les autres modes de rupture du contrat de travail ${ }^{1}$. Ce dernier peut prendre fin soit par extinction, soit par résiliation.

L'extinction du contrat de travail, en vertu de la loi, ne dépend pas directement de la volonté des parties. C'est un fait juridique: abandon du travail, absence d'au moins trois mois du travailleur causée par une détention provisoire (toutefois l'établissement peut résilier sans préavis le contact de travail du fait de l'infraction), la mort du travailleur, etc. Est abandon du travail le cas où le travailleur sans autorisation s'abstient d'accomplir son travail ou le fait de ne pas se présenter au travail sans informer l'établissement, dans le délai prescrit, des raisons de l'absence (le premier jour de son absence et au plus tard le lendemain).

La résiliation du contrat de travail se réalise d'une part d'un commun accord des volontés des parties: la résiliation du contrat de travail en vertu d'un accord entre les parties (art. 30, 1 er, al. 1 du Code du travail), la résiliation du contrat de travail à l'expiration de la période pour laquelle il a été conclu ou le jour de l'achèvement du travail pour l'accomplissement duquel il a été conclu (art. 30, 1 er, al. 4 et 5 du Code du travail); d'autre part, par déclaration unilatérale de volonté: démission du travailleur ${ }^{2}$ avec préavis (art. 30, 1er, al. 2 et art. 32-37 du Code du travail) ou sans préavis (art. 55 et art. 57 qui se rapporte à l'art. 48 du Code) et licenciement du travailleur, résiliation du contrat de travail avec ou sans préavis.

Toute distinction entre ouvriers et employés notamment au regard de la résiliation du contrat de travail a été abolie par le Code du travail du 26 juin 1974 qui est entré en vigueur le 1 er janvier 1975 . Un système unique des droits et devoirs des travailleurs est adopté.

- PLISZKIEWICZ, M., Institut de l'État et du Droit de l'Académie polonaise des sciences, Varsovie, Pologne.

1 D'autres sources que le contrat de travail peuvent être à la base du rapport du travail; ces situations ne seront pas examinées ici.

2 Le problème de la démission n'est pas abordé dans cet article. 
La caractéristique fondamentale de la réglementation juridique antérieure était la liberté totale de résiliation avec préavis des contrats de travail conclus pour une période d'essai ou pour une durée indéterminée. Chaque partie pouvait résilier unilatéralement un tel contrat sans avoir à indiquer les motifs de sa décision.

La jurisprudence polonaise précédant la codification a admis que la résiliation d'un contrat de travail à durée indéterminée n'était pas laissée à la libre décision de l'établissement mais qu'elle devait être conforme au but socio-économique du droit et rester en accord avec les règles de vie en so-

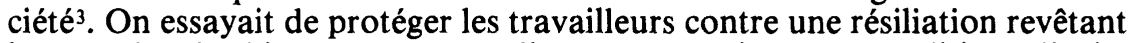
le caractère de chicane et contre celle portant atteinte aux conditions d'existence du travailleur et de sa famille, en se référant aux clauses générales de l'art. 5 du Code civil ${ }^{4}$. En vertu de cette disposition une action ou une abstention contraire au but socio-économique du droit ou aux règles de vie en société n'est pas considérée comme exercice de ce droit et ne jouit pas de protection légale.

La décision de licenciement est prise par le chef d'établissement conformément au principe de la direction unipersonnel déterminé dans le préambule et dans l'art. 4 du Code du travail.

La résiliation du contrat de travail (avec ou sans préavis) est effectuée par écrit et comprend une information détaillée sur les moyens juridiques dont dispose le travailleur (art. 30, $2^{\mathrm{e}}$ et $3^{\mathrm{e}}$ du Code du travail).

\section{LA RESILIATION DU CONTRAT DE TRAVAIL AVEC PREAVIS PAR L'ÉTABLISSEMENT}

Sont concernées d'une part, la rupture du contrat de travail à durée indéterminée, d'autre part, la rupture des contrats de caractère «temporaire»: période d'essai, période de stage préliminaire ${ }^{5}$ et - sous certaines conditions - contrat à durée déterminée.

\section{Période d'essai et période de stage préliminaire}

La résiliation s'effectue normalement à l'expiration de leur durée mais ils peuvent être résiliés avant terme moyennant un préavis (art. $30,2 \mathrm{e}$ du Code du travail). Ce dernier en ce qui concerne la période d'essai est de trois jours ouvrables, lorsque le contrat ne dépasse pas deux semaines et de deux semaines, lorsque le contrat ne dépasse pas trois mois 6 . Et en ce qui concerne le stage préliminaire, le préavis est de deux semaines.

3 ZIELINSKI, V. Tadeusz, «La dissolution du rapport du travail», Droit Polonais Contemporain (version française), no 2, 1976, p. 20 et la littérature citée dans cet article.

4 Cette disposition a été transposée dans l'art. 8 du Code du travail.

5 Le contrat de stage préliminaire, non exclusif du contrat à l'essai est obligatoire dans deux situations: travailleur occupant un emploi pour la premiere fois (sauf exceptions) et travailleur non employé au moins une année dans un établissement (l'art. $28, \quad 1^{\text {er }}$ du Code $d u$ travail).

6 La période d'essai ne dépassant pas trois mois est prévue s'il s'agit d'un travailleur à un poste de direction ou autre poste indépendant ou encore à un poste comportant la responsabilité matérielle pour les biens qui lui sont confiés (l'art. 27 du Code du travail). 
Il peut être résilié avant l'expiration de la période pour laquelle il a été conclu pourvu qu'il l'ait été pour une période de plus de six mois et que les parties aient prévu cette faculté, moyennant un préavis de deux semaines (art. 33 du Code du travail). Cet article a un caractère dispositif.

En cas de la résiliation avec préavis du contrat de travail pour une période d'essai, pour une période de stage préliminaire et à durée déterminée le Code du travail n'exige pas de contrôle syndical.

La résiliation du contrat de travail à durée indéterminée

Elle est soumise à des restrictions.

Le chef d'établissement ne peut notifier le préavis qu'après consultation des organes compétents des syndicats. Le chef d'établissement qui a l'intention de résilier avec préavis un contrat de travail à durée indéterminée, en informera par écrit le conseil d'entreprise en indiquant en même temps la cause justifiant le licenciement. Lorsque le conseil d'entreprise estime que le licenciement serait injustifié, il peut, dans les cinq jours suivant la notification en question, présenter par écrit au chef d'établissement ses réserves motivées. $S^{\prime} i l$ ne retient pas les réserves présentées dans le délai prescrit, le chef d'établissement est tenu de soumettre le cas au président de l'instance syndicale immédiatement supérieure par rapport au conseil d'entreprise, qui peut se prononcer sur les réserves dans les cinq jours suivant le moment où il a été saisi. Après avoir examiné l'avis du président de cette instance syndicale, ainsi que dans le cas où ce dernier ne prend pas position dans le délai ci-dessus, le chef d'établissement prend sa décision au sujet du licenciement (art. 38 du Code du travail).

Cette procédure est, dans son intégralité, la condition nécessaire de la faculté de dénonciation du contrat de travail à durée indéterminée. Aussi l'établissement de travail n'a-t-il aucun droit à notifier le préavis avant l'expiration des délais prévus pour l'examen par les instances syndicales compétentes. "Il ne peut alors être question d'une suspension du droit de dissoudre un rapport de travail, car ce droit ne prend naissance qu'à la fin de la procédure prévue à l'art. 38 du Code du travail»?

Les réserves formulées par le conseil d'entreprise ou par le président de l'instance syndicale supérieure n'empêchent pas juridiquement l'établissement de licencier. Il s'agit seulement de mesures visant à faire renoncer le chef d'établissement à son intention de résilier le contrat ${ }^{8}$.

L'inobservation de ces dispositions n'entraîne pas la nullité du préavis. En l'absence d'un recours du travailleur le contrat sera résilié à l'expiration du préavis.

7 ZIELINSKI, V.T., op. cit., p. 22.

8 ZIELINSKI, V.T., op. cit., p. 22 et Maria MATEY, «La protection des travailleurs contre le licenciement en Pologne», Droit Social, no 2, 1980, p. 198. 
En cas de non respect des dispositions légales de procédure (consultation syndicale, forme, délais, etc.) un recours est ouvert au travailleur.

$L$ a résiliation ne peut prendre effet qu'après respect du délai de préavis, qui varie selon l'ancienneté du salarié dans l'établissement; toutefois des exceptions sont prévues par la loi ${ }^{9}$.

Ces délais sont: de deux semaines, si le travailleur a été employé pendant moins d'un an; - d'un mois, si le travailleur a été employé au moins un an; - de trois mois, si le travailleur a été employé au moins dix ans.

Le préavis se termine un samedi lorsque le délai de préavis est de deux semaines, ou le dernier jour du mois dans les autres cas.

Ces délais ne s'appliquent pas lorsque le contrat est résilié en vertu d'un accord des parties qui peuvent alors librement fixer le délai de résiliation.

Tout le licenciement doit avoir une cause le justifiant. Ce principe est d'application générale. C'est un abandon de la conception traditionnelle de la résiliation considérée comme un acte abstrait. Ces causes peuvent exister soit du côté de l'établissement, soit du côté du travailleur.

Dans tous les autres cas, le licenciement est injustifié et peut être contesté par le travailleur. L'existence de causes justifiées de la résiliation est une condition de fond, qui restreint au sens juridique (et non seulement au sens pratique, comme les réserves syndicales) la liberté de licenciement ${ }^{10}$.

Il n'existe pas (sauf exceptions) ${ }^{11}$ d'énumération légale des causes justificatives. Le législateur s'est contenté d'insérer dans le code une disposition selon laquelle la commission de recours pour les questions du travail déclare la notification du préavis sans effet, et si le contrat de travail a déjà pris fin, elle prononce la réintégration du travailleur dans l'emploi aux conditions antérieures, si elle établit son caractère injustifié (art. 45 du Code du travail). Il faut donc tenir compte des circonstances de chaque cas d'espèce.

Dans la doctrine on souligne qu'est injustifié tout licenciement contraire au but socio-économique de ce droit. C'est le cas par exemple pour un travailleur qui remplit dûment ses devoirs, ou lorsque la résiliation n'est pas motivée par les nécessités d'une politique rationnelle de l'emploi découlant des changements dans l'organisation du travail ou de la production. Certains auteurs entendent de façon plus vaste encore la notion du licenciement injustifié. On exprime l'opinion que cette notion renferme égalernent des éléments des règles de vie en société ${ }^{2}$. Mais le licenciement d'un travailleur qui, sans raison, ne se conforme pas aux instructions de son superieur ne contrevient pas aux règles de la vie en société en République Populaire de

9 V. l'art. 36, $4^{\mathrm{e}} \mathrm{du}$ Code du travail et règlement du Conseil des ministres du 20 septembre 1974 concernant la durée des emplois antérieurs qui entrent dans le calcul de l'ancienneté dont dépend le délai de préavis (Journal des Lois, no 37, 1974, texte 216).

10 ZIELINSKI, V.T., op. cit., p. 22.

11 V. l'art. 196 du Code du travail qui prévoit l'énumération des causes justificatives de la résiliation du contrat de formation professionnelle de l'adolescent.

12 ZIELINSKI, V.T., op. cit., p. 23 et la littérature citée dans cet article. 
Pologne ${ }^{13}$. Est protégé contre le licenciement seulement le travailleur qui accomplit consciencieusement son travail ${ }^{14}$.

La Cour suprême a donné l'explication plus générale de la cause justifiant le licenciement avec préavis dans deux arrêts suivants:

- Pour juger de la cause justifiant le licenciement avec préavis il faut prendre en considération les intérêts de l'établissement et ceux du travailleur ayant un lien avec le but et la véritable nature du rapport de travail, notamment, les devoirs des parties tels qu'ils sont déterminés par le Code du travail (voir surtout art. 10, 1 er et art. 12 du Code du travail 15 ).

- La commission de recours et, en deuxième instance, le tribunal du travail et d'assurances sociales, doit prendre en considération toutes les circonstances ayant pu justifier le licenciement avec préavis et non pas seulement la cause indiquée par l'établissement, laquelle reste toujours essentielle, mais aussi toutes les circonstances professionnelles (mode de travail, ancienneté) et extraprofessionnelles relatives au travailleur licencié ${ }^{16}$.

Dans la pratique judiciaire polonaise, telle qu'elle s'est développée au cours des cinq ans qui se sont écoulés depuis l'entrée en vigueur du Code, l'opinion s'est établie selon laquelle n'est pas «injustifiée»» la résiliation avec préavis invoquant: a) l'inaptitude du travailleur à un travail donné; b) une conduite répréhensible obstinée du travailleur; c) d'importantes causes techniques ou organisationnelles surgissant du côté d'employeur (réorganisation, réduction d'emplois, etc.). En revanche, est considérée comme «injustifiée» la résiliation avec préavis malgré des circonstances sociales militant contre le licenciement d'une personne travaillant consciencieusement dans les limites de ses possibilités (cela concerne notamment les femmes soutiens de famille et les travailleurs justifiant d'un long stage) ${ }^{17}$.

\section{La protection spéciale contre le licenciement avec préavis}

Indépendamment de la protection générale relative à la stabilité du rapport de travail, le droit polonais prévoit aussi une protection spéciale, laquelle consiste à restreindre la faculté de résiliation par l'établissement des contrats de travail de certains groupes de travailleurs pour lesquels la rési-

13 L'Arrêt de la Cour suprême du 13 juin 1973 - I PR 160/73, Orzecznictwo Sadu Najwyzszego, (La jurisprudence de la Cour suprême) 1974, texte 75.

14 L'Arrêt de la Cour suprême du 13 janvier 1975 - III PRN 67/74 cité dans Kodeks pracy $z$ Komentarzem (Code du travail avec commentaires), un ouvrage collectif sous la direction de Mieczystaw PIEKARSKI, Varsovie, 1979, p. 105.

15 L'Arrêt de la Cour suprême du 10 septembre 1975 - I PRN 34/75, Code du travail avec commentaires, op. cit., p. 105.

16 L'Arrêt de la Cour suprême du 19 juillet 1976 - I PZP 29/76, Code du travail avec commentaires, op. cit., p. 105.

17 MATEY, V. Maria, op. cit., p. 197. 
liation du contrat de travail avec préavis est prohibée. Il s'agit aussi bien des raisons d'ordre personnel et familial que de l'exercice de fonctions sociales ou publiques.

Dans le premier groupe on classe: les congés payés, l'absence justifiée, les deux dernières années précédant l'âge de la retraite, (à condition toutefois que la période d'emploi lui permette de faire valoir ses droits à la retraite lorsqu'il aura atteint cet âge), la grossesse et le congé de maternité, le congé sans solde de 3 ans pour élever un enfant jusqu'à ce que l'enfant ait 4 ans révolus, le service militaire du travailleur, le service militaire du mari de la travailleuse, le soupçon que le travailleur est porteur d'une maladie contagieuse, la maladie contagieuse d'un travailleur du service de santé et la convocation au travail pour combattre une épidémie.

Le second groupe des circonstances comprend l'exercice des fonctions de délégué syndical, d'inspecteur social du travail, de membre de la commission d'arbitrage à l'intérieur de l'établissement ainsi que des fonctions électives dans une organisation sociale, à condition que le travailleur bénéficie d'un congé sans solde durant l'exercice de ses fonctions.

\section{Le recours contre le licenciement avec préavis}

Tout travailleur peut former un recours contre le licenciement avec préavis devant la commission de recours pour les questions du travail.

Ce recours est conçu en droit polonais comme un acte juridique qui n'a pas besoin d'être motivé. Il n'est donc pas nécessaire de donner des faits concrets visant à nier le bien-fondé du licenciement avec préavis. En revanche, l'établissement se voit obligé, quant à lui, de prouver devant la commission de recours pour les questions du travail le bien-fondé du licenciement. Le travailleur peut former un recours contre son licenciement avec préavis indépendamment de l'avis du conseil d'entreprise, même s'il n'a pas présenté au chef d'établissement de réserves motivées quant au licenciement envisagé.

Lorsque la commission de recours constate que la résiliation avec préavis du contrat de travail à durée indéterminée est injustifiée ou bien lorsqu'elle constate que la signification du préavis a été faite en violation des dispositions relatives au licenciement avec préavis, elle déclare la notification du préavis sans effet. Mais, si le contrat de travail a déjà été résilié, elle prononce la réintégration du travailleur dans l'emploi aux conditions antérieures.

La décision prononçant la réintégration du travailleur dans l'emploi est absolument obligatoire pour l'établissement. Si le travailleur se présente au travail dans les sept jours à compter du moment où la décision est passée en force de chose jugée, l'établissement ne peut refuser de l'employer aux conditions antérieures. L'établissement ne peut non plus se libérer de cette obligation en payant une indemnité18. 


\section{LA RESILIATION DU CONTRAT DE TRAVAIL SANS PREAVIS PAR} L'ETABLISSEMENT

Le licenciement sans préavis exige la forme écrite (art. 30, 3 du Code du travail). L'établissement est tenu d'indiquer, en outre, par écrit, la cause justifiant ledit licenciement (art. 54 du Code du travail).

Le licenciement sans préavis est possible uniquement dans les cas prévus par le Code du travail. Celui-ci indique deux types de causes justifiant la résiliation du contrat de travail sans préavis: la faute du travailleur et des causes indépendantes de la faute du travailleur.

\section{Le licenciement sans préavis suite à la faute du travailleur}

L'établissement peut résilier un contrat de travail sans préavis suite à la faute du travailleur pour violation par ce dernier de ses devoirs fondamentaux, plus particulièrement le fait d'avoir perturbé l'ordre et la tranquillité sur le lieu de travail, l'absence injustifiée au travail, le fait de s'être présenté au travail en état d'ébriété ou d'avoir consommé de l'alcool pendant les heures de travail ainsi que d'avoir abusé intentionnellement des prestations de caractère social (art. 52, 1, al. 1 du Code du travail).

Les dispositions concernant le licenciement sans préavis n'énumèrent que des exemples en ce qui concerne les devoirs fondamentaux du travailleur. La doctrine en droit du travail souligne que tous les devoirs du travailleur indispensables à l'accomplissement d'un travail déterminé, et, notamment, ceux qui sont énumérés à l'article 100 du Code du travail et au 5 du Règlement du Conseil des Ministres sur le règlement intérieur du travail ${ }^{19}$, sont des devoirs fondamentaux. Cette opinion est partagée par la jurisprudence de la Cour suprême. L'observation des règles relatives à la sécurité et à l'hygiène du travail et à la prévention des incendies, l'observation des dispositions relatives aux secrets d'États et aux secrets internes au service ainsi que la protection des biens de l'établissement font partie des devoirs fondamentaux des travailleurs ${ }^{20}$. L'inexécution des instructions du supérieur qui perturbent l'ordre et la discipline dans le travail justifie le licenciement sans préavis pour violation grave par le travailleur de ses devoirs fondamentaux ${ }^{21}$.

La critique des dirigeants de l'établissement, si elle est non fondée, malicieuse ou exprimée de manière inconvenante peut être considérée comme une violation grave des devoirs fondamentaux ${ }^{22}$. pour:

L'établissement peut également licencier un travailleur sans préavis

19 Journal des Lois, no 49, 1974, texte 299, avec amendements postérieurs.

20 L'Arrêt de la Cour suprême du 22 septembre 1976 - I PRN 62/76, Code du travail avec commentaires, op. cit., p. 131.

21 L'Arrêt de la Cour suprême cité ci-dessus, v. note 13.

22 L'Arrêt de la Cour suprême du 29 janvier 1975 - III PRN 69/74, La jurisprudence de la Cour suprême, op. cit., 1975, texte 124 . 
- une infraction commise par celui-ci au cours de son contrat de travail, rendant impossible le maintien dans l'emploi qu'il occupe, pour autant que cette infraction est évidente ou a été constatée par un jugement passé en force de chose jugée,

- la perte par la faute du travailleur, de ce qui est exigé pour être employé au poste qu'il occupe (par exemple retrait du permis de conduire) (art. 52, 1 , art. 2 et 3 du Code du travail).

Le contrat de travail ne peut être résilié sans préavis suite à la faute du travailleur que pendant un mois à partir du moment où l'établissement a pris connaissance du fait pouvant justifier une telle résiliation.

\section{Le licenciement sans préavis pour des causes indépendantes de la faute du travailleur}

Parmi ces causes, il faut énumérer: l'incapacité de travail consécutive à une maladie dont la durée dépasse les périodes prévues par le Code du travail ainsi que l'absence justifiée au travail de plus d'un mois pour d'autres causes que la maladie.

La période de protection du travailleur contre le licenciement sans préavis dépend de l'ancienneté des travailleurs dans l'établissement (art. 53,

$1 \mathrm{du}$ Code). Si le travailleur est employé dans un établissement depuis moins de six mois, la résiliation du contrat peut intervenir lorsque l'incapacité de travail pour cause de maladie dure plus de trois mois. En revanche, si le travailleur est employé dans un établissement depuis plus de six mois au moins ou si l'incapacité de travail résulte d'un accident de travail ou d'une maladie professionnelle, l'établissement de travail peut résilier le contrat sans préavis seulement lorsque l'incapacité de travail excède la période couverte par l'allocation-maladie. Cette période ne peut dépasser six mois ou neuf mois en cas de tuberculose. À la fin de cette période, lorsque le travailleur garde une incapacité de travail pour cause de maladie, mais que la poursuite du traitement médical ou la rééducation laissent espérer que le travailleur retrouvera sa capacité de travail, le service de l'allocation peut être porté à neuf mois ou douze mois en cas de tuberculose.

Le contrat de travail ne peut être résilié sans préavis pour cause de maladie même après l'expiration de cette période (couverte par l'allocationmaladie) lorsque la cause de l'absence a disparu et que le travailleur s'est présenté au travail.

L'établissement doit, dans la mesure du possible, réemployer le travailleur qui, dans les six mois suivant la résiliation sans préavis, se présente à l'établissement quand il est définitivement guéri (art. 53, 5 du Code).

Le contrat de travail ne peut être résilié sans préavis lorsque l'absence du travailleur est causée par la garde d'un enfant ou l'isolement du fait d'une maladie contagieuse, dans la période couverte par l'allocation à ce titre. 
Le contrôle syndical du licenciement sans préavis

Le licenciement sans préavis soit pour faute soit pour des causes indépendantes de la faute, ne peut être effectué qu'après un contrôle syndical ayant un caractère consultatif. En effet, le chef d'établissement ne prendra la décision de résilier le contrat de travail sans préavis qu'après avoir demandé l'avis au conseil d'entreprise qu'il informe du motif justifiant la résiliation du contrat. Si le bien-fondé de la résiliation suscite des réserves de la part du conseil d'entreprise, celui-ci exprime son avis dans un délai de trois jours au plus.

Les moyens juridiques dont dispose le travailleur en cas de violation des dispositions relatives à la résiliation du contrat sans préavis

Le travailleur dont le contrat de travail a été résilié sans préavis en violation des dispositions relatives à la résiliation du contrat a le droit d'exiger soit d'être réintégré dans son travail aux conditions antérieures soit de bénéficier d'une indemnité. La décision de réintégrer ou d'indemniser incombe à la commission de recours pour les questions du travail statuant en $1^{\mathrm{re}}$ instance, et au tribunal du travail et d'assurances sociales, en $2^{\mathrm{e}}$ instance.

\section{Les réclamations à l'arbitrage de dommages résultant de la violation d'une clause de renonciation à la grève}

\section{Claude D'Aoust}

La Cour suprême du Canada vient de rendre une décision qui affermit singulièrement l'autorité de l'arbitre de grief québécois'. Elle poursuit ainsi dans le sillage de l'affaire G.M. c. Brunet tranchée il y a quelques années'.

Dans ce dernier cas, il avait été décidé que la définition laconique du grief du Code du travail québécois ${ }^{3}$, par comparaison à celle de la loi ontarienne par exemple ${ }^{4}$, ne pouvait justifier de subtiles distinctions destinées à

- D’AOUST, Claude, professeur agrégé, École de relations industrielles, Université de Montréal.

1 Shell Canada Ltd. c. Travailleurs unis du pétrole du Canada, Local 1 et al, jugement unanime de la Cour suprême du Canada, le 27 juin 1980, rédigé par le juge Chouinard.

2 General Motors of Canada Ltd. c. Pierre Brunet et al, [1977] 2 R.C.S. 537.

3 Code du travail, L.R.Q. 1977 c. C-27. L'article 1 définit le grief comme «toute mésentente relative à l'interprétation ou à l'application d'une convention collective».

4 The Labour Relations Act, R.S.O. 1970, c. 232. L'article 37 englobe dans la notion de grief «tout différend découlant de l'interprétation, de l'application, de l'administration ou de la prétendue violation de la convention collective» (Traduction et adaptation de l'auteur). 